\title{
INTERNET OF THINGS AND PRIVACY
}

\author{
Valentina Amenta ${ }^{1}$, Maria Claudia Buzzi ${ }^{1}$, Marina Buzzi ${ }^{1}$ and Antonella Montemurro ${ }^{2}$ \\ ${ }^{l} \mathrm{CNR}-$ IIT, Italy \\ ${ }^{2}$ University of Pisa, Italy
}

\begin{abstract}
Today the Internet of Things (IoT) empowers our lives, simplifying interaction and services in many fields including smart homes and cities, telemedicine and healthcare, transportation, security and social life. In many IoT applications, personal data are automatically collected and stored in cloud systems, processed and used for purposes such as creating user profiles, monitoring health, personalized advertising and more. The main cost of this process involves data that are collected by providers and organizations. This paper discusses important privacy and security issues raised by the main IoT devices, specifically considering the obligations imposed by the General Data Protection Regulation (GDPR) on the organizations collecting data relating to people in the European Union.
\end{abstract}

\section{KEYWORDS}

Privacy, Security, Internet of Things (IoT), GDPR

\section{INTRODUCTION}

Digital services are now increasingly embedded in the surrounding environment, simplifying and transforming our lives. This process is so pervasive that it is impossible today to think of a world without the Net. Objects too are connected, creating the so-called Internet of Things (IoT), a system of linked devices or smart objects characterized by a unique identifiers able to transfer data through the Internet without human intervention. Soon we will be totally in thrall to our objects. Future scenarios will include self-driving cars, fridges writing the shopping list, thermostats adjusting temperature according to our needs, elderly and sick people being monitored using sensors, smart watches and other wearable devices. All these services aim to make our life simpler, safer and more comfortable. This will undoubtedly bring many benefits, but also presents some challenges, especially from a legal point of view. The main cost is no longer monetary but rather concerns data (sometimes sensitive), which can be collected by providers and organizations with or without the user's permission. Are we really willing to relinquish our "digital life" to the Internet clouds in exchange for a simpler life? Are we ready for these changes or do we need a greater awareness of what looms in the near future? This paper presents some privacy and security issues raised by IoT, specifically considering the obligations stated by the General Data Protection Regulation (GDPR) (679/2016 https://gdpr.eu/) on the organizations collecting data regarding people in the European Union (EU). GDPR has profoundly changed the world of the IoT, and not only in Europe, with obligations such as more information, transparency and control over the user data as well as adopting suitable security measures to prevent and contain data breaches.

\section{IOT DEVICES AND APPLICATIONS}

IoT is a technological evolution of the Internet in which objects are given an identity through the association of a unique identification and can sense, keep and transmit data detected from the external environment. Well-known examples of IoT devices are wearable items, such as watches, bracelets, rings, glasses, cams, visors and RFID tags implanted under the skin. Examples of privacy issues for smart glasses are introduced in (Delail \& Yeun, 2015). Google Glass components (GPS, microphone and micro-camera) enable face detection and allows taking pictures and videos. However, people should not record videos on certain private occasions or use it as a tool to monitor people in the vicinity (Chang et al., 2019). In 2013, some Privacy Guarantors sent 
a letter to Google expressing their concerns about privacy regarding the development of Google Glass. ${ }^{1}$ Although so far there are few elements that prove the existence of effective discrimination, the data collected by intelligent objects can be a revenue optimization tool for personalized pricing based on detected behaviors (European Data Protection Supervisor, 2015). An example is the cost of a life insurance policy, which can depend on a user's habits regarding physical (in)activity, smoking, health issues, etc. Amazon smart glasses (Echo Frames) are simpler than Google Glass but they interact with Alexa, the Amazon virtual assistant, thus listening continuously and capturing information about the user. Furthermore, to make phone calls, the user must share their contact list with Amazon (Smith, 2019).

Smart wristbands allow monitoring physical activities and physiological parameters. Producers could introduce a de-identification of the data to share by removing information useful for identification. However, this may not be sufficient due to the ease of data re-identification: for instance, each of us has a unique gait. This means that if we know the gait and the habits of a user, it would be possible to identify that individual among millions of anonymized data belonging to all the bracelet users, and the presence of data geolocation could pose privacy risks. Furthermore, collected data can be shared on social networks; this can lead to privacy violations, e.g., in the involuntary publication of the activity. Some trackers also transmit personal data and even private address lists to servers. According to Article 7 of Regulation 679/2016 the user must consent to data processing.

A 2016 review of smart watches highlighted the need for studies to identify solutions related to privacy, confidentiality and security of smart watch data, as well as the ethics of data ownership and use (Reeder \& David, 2016). Also, a recent paper analyzing the usefulness of smart watch electrocardiograms highlighted concerns about the safety and security of using direct-to-consumer smart watches in healthcare; companies own the raw data and the user has no control over the use of their information. In addition, although data are stored anonymously, based on user activity and location "digital traces" may still reveal an individual's identity (Isakadze \& Seth, 2019). Other examples of wearable devices are smart rings for health/fitness tracking and payments, and what was previously stated for wristbands and smart watches also applies to these; in addition, school uniforms with tracking chips have been introduced in China, ${ }^{2}$ where embedded cameras can record the movement of students in school for security reasons, violating the students' privacy (Chang et al., 2019).

Regarding non-wearable devices, sensors are widely used for improving the user experience at home and at work, monitoring environmental parameters such as light, humidity, movement, position, etc. They should minimize tracking, storage and sharing of personal and sensitive data. The same considerations can also apply to various tools, such as mobile devices and robots, which can monitor people's behavior and data.

A very useful and popular ${ }^{3}$ solution is the voice assistant such as Amazon's Alexa and the Google Home (Lei et al., 2017), "Ready On" devices controlled by vocal interaction. However, this mechanism raises serious doubts about user privacy: vocal conversations are stored to be processed, and may be manually revised for product development purposes. In response to growing user concerns about privacy, Amazon has created a webpage for Alexa users to control how their personal data is collected, saved and processed, and configure his/her privacy preferences. These devices have also revealed vulnerabilities. A study carried out on Amazon's Alexa (applicable to Google Home as well) found that anyone who pronounces the word "Alexa" correctly can access the services, thus highlighting a lack of safe authentication. This vulnerability allows external parties to "manipulate" the home from outside; intruders can activate TV content, make purchases, take control of the electrical system, or even unlock smart locks. To counter this, one proposal is to make the system safer with command validation that works only if the user is close to the device.

An important area of IoT is medicine. Wearable devices enable healthcare workers to accurately evaluate and monitor patients in their daily life, allowing optimization of the treatment and facilitating disease research. An example is the treatment of Parkinson's disease via wireless body sensors that allow a virtual evaluation of the patient, allowing the doctor to verify the therapy's effectiveness (Zhan et al., 2018). The use of artificial intelligence for big data analysis and decision support systems applied to large shared medical datasets and user-generated content through wearable devices has great potential but also leads to concerns over privacy, such as user trust, data privacy, transparency over the control of data ownership, and the implications of data analytics regarding personal privacy.

\footnotetext{
${ }^{1}$ https://epic.org/privacy/google/glass/default.html

${ }^{2} \mathrm{https}$ //www.thesun.co.uk/news/8056887/china-tracking-chips-school-uniforms/

${ }^{3} \mathrm{https}$ //www.statista.com/statistics/973815/worldwide-digital-voice-assistant-in-use/
} 
Smart toys, such as conversational dolls like "Hello Barbie" or "My Friend Cayla" pose privacy problems. "My Friend Cayla" has a microphone and an Internet connection without any security measures. It has been hacked to make it whisper ugly words and steal sensitive data (Taylor \& Michael, 2016). Germany has expressly prohibited its sale, classifying the doll as an illegal espionage tool (intercepting log data, audio, date of birth, gender and children's preferences) (McReynolds et al., 2017). In the same way, "Hello Barbie" records what children say and parents can listen and share children's thoughts on social media. The manufacturer claimed that collected data are only used to improve their algorithms. However, this data can be shared with other server providers. There is a strong contrast between people who consider this feature innovative and those who consider it a threat to children's rights to privacy, since playing is a way to discover the world and children are unaware that someone is actually listening to them (Taylor \& Michael, 2016). However, smart toys can also be helpful for children and their families, e.g., by using them for early diagnosis. A recent work (Bondioli et al., 2020) describes an IoT system created to automatically identify the movements applied to the toys by a child in order to diagnose autistic spectrum disorders at an early stage. Another example is the "CareToys" which deals with early disability diagnosis in premature infants. The system proposes exercises based on the level and needs of the child. Results confirmed that it is possible to reduce the time of diagnosis from two years to a few months, anticipating the rehabilitation process (Sgandurra et al., 2017).

RFID implants can speed up daily actions, such as paying, opening doors, computer/device authentication, starting the car, or obtaining rapid access to the emergency room. There are no studies that evaluate their actual danger in terms of personal health since there are no examples of adequate experimentation before marketing. However, the risks of any implant include infection, pain, lesions at the puncture site, local anesthetic and allergic reaction (Wilson et al., 2019). Furthermore, critical privacy concerns include the protection of personal privacy, the digital divide gap (services cannot be exploited by people who are not connected) and data security. If the RFID chip is hacked, the person is exposed to serious risks, such as money being stolen, personal information accessed, etc. Moreover, if the chip incorporates a GPS, the person's movements could be constantly monitored and thus they can be stalked (Levine et al., 2007). According to European legislation, the chip implant equipped with GPS is illegal in all European countries. A recent study with 453 participants (small-business owners) in Australia, India, UK and the US showed that the perceived reasons for rejecting an employee ID implant clearly outnumbered those for accepting it, for various reasons or points of view (Michael et al., 2017): Inhumanity, Intrusiveness (Invasion and Violation) -- Physical and Psychological, Invasion of Privacy, Invasion of Human Rights, Cultural Issues, Health Issues, Cosmetic Issues, Unwarranted.

\section{CONCLUSION}

The IoT opens up new vistas for everyday applications in innumerable areas. Technologies such as RFID, sensors and wearable devices collect an enormous amount of data. Many people are entrusting their personal data to cloud services but data breaches are very frequent and on the rise: in the first 6 months of 2019 there were more than 3,800 publicly disclosed breaches exposing 4.1 billion compromised records. ${ }^{4}$

Processing of data to be counted as profiling must have three essential elements: 1) imply some form of automation, 2) have personal data as object and 3) be addressed to personal aspects of the person. Based on the general principles of Article 5 of the GDPR, the data must be processed lawfully, correctly and transparently. They must be collected and processed for explicit and compatible purposes, and they must be adequate and exact with respect to the treatment. They must be kept for a suitable time and treated securely. The consent of the interested party is mandatory: there is an absolute ban on processing sensitive personal data without consent. Personal data, often requested to download apps, is collected and stored in databases for commercial purposes and might be sold to third parties. This can happen without the user's knowledge, taking advantage of extraterritorial servers, which hamper the enforcement of rules to protect privacy (Visconti, 2020). Indeed, the emergence of cloud solutions makes it very difficult to control how user data are really utilized and exploited over time. Large companies are often located outside Europe, so different legislations may be applied. Some threats derive from how the company manages data preservation, but also from possible security breaks and stealing data. Since it is very difficult to have a system resistant to any new security attack, it is clear that 
by providing consensus to the security and privacy policies of the providers, the user accepts part of this risk. A possible solution for mitigating risks that arise from data storage on centralized cloud systems is to keep and process data locally applying Fog and Edge computing (Yousefpour et al., 2019), e.g., by having IoT modular plug and play objects that communicate via Wi-Fi o BLE (Bluetooth Low Energy) protocols and managing their interaction control and data storage locally, enabling users to have more control of their data.

Fundamental rights to privacy and the protection of personal data enshrined in EU treaties and the Charter of Fundamental Rights are very important for the protection of human dignity. Therefore, the principles of data protection require a new way of thinking by combining ethical principles (European Data Protection Supervisor, 2015).

\section{REFERENCES}

Bondioli, M., Chessa, S., Narzisi, A., Pelagatti, S., and Piotrowicz, D., 2020. Capturing Play Activities of Young Children to Detect Autism Red Flags. In ISAmI 2019, 10th International Symposium on Ambient Intelligence, 71-79

Chang, V., Xu, X., Wong, B., Mendez, V., 2019. Ethical Problems of Smart Wearable Devices. 4th International Conference on Complexity. In Proc. of the 4th International Conference on Complexity, Future Information Systems and Risk, Elsevier. 121-129

Delail, B. A., Yeun, C. Y., 2015. Recent advances of smart glass application security and privacy. In Proceedings of the 10th International Conference for Internet Technology and Secured Transactions (ICITST). 65-69

European Data Protection Supervisor, Opinion 4/2015: “Towards a new digital ethics", 11 Sept 2015, https://edps.europa.eu/sites/edp/files/publication/15-09-11_data_ethics_en.pdf

Isakadze, N., Seth, S. M., 2019. How useful is the smartwatch ECG? In Trends in Cardiovascular Medicine, Volume 30, Issue 7, October 2020, 449-450, https://doi.org/10.1016/j.tcm.2019.10.010

Lei, X., Tu, G. H., Liu, A. X., Ali, K., Li, C. Y., and Xie, T., 2017. The Insecurity of Home Digital Voice Assistants--Amazon Alexa as a Case Study. In Cryptography and Security, arXiv:1712.03327 https://arxiv.org/abs/1712.03327

Levine, M., Adida, B., Mandl, K., Kohane, I., and Halamka, J., 2007. What are the benefits and risks of fitting patients with radiofre-quency identification devices? In PLoS medicine, 4(11), e322

McReynolds, E., Hubbard, S., Lau, T., Saraf, A., Cakmak, M., and Roesner, F., 2017. Toys that listen: A study of parents, children, and internet-connected toys. In Proc. of CHI 2017 Conference. 5197-5207

Michael, K., Aloudat, A., Michael, M. G., and Perakslis, C., 2017. You Want to Do What with RFID? Perceptions of radio-frequency identification implants for employee identification in the workplace. In IEEE Consumer Electronics Magazine, 6(3), 111-117.

Reeder, B., and David, A., 2016. Health at hand: A systematic review of smart watch uses for health and wellness. In Journal of Biomedical Informatics 63. 269-276

Sgandurra, G., Lorentzen, J., Inguaggiato, E., Bartalena, L., Beani, E., Cecchi, F., Dario, P., Giampietri, M., Greisen, G., Herskind, A., Nielsen, J. B., Rossi, G., Cioni, G., CareToy Consortium. 2017. A randomized clinical trial in preterm infants on the effects of a home-based early intervention with the CareToy System. In PloS one, 12(3):e0173521.

Smith, D. 2019. Amazon Echo Frames -- here's what you didn't know about Amazon's new smart glasses. https://www.cnet.com/how-to/amazon-echo-frames-heres-what-you-didnt-know-about-amazons-new-smart-glasses/

Taylor, E., Michael, K., 2016. Smart Toys that are the Stuff of Nightmares. In IEEE Technology and Society Mag. 8-10.

Visconti, R. M., 2020. Big Data Valuation. In The Valuation of Digital Intangibles. Palgrave Macmillan, Cham. 345-360

Wilson, R. P., Börger, L., Holton, M. D., Scantlebury, D. M., Gómez-Laich, A., Quintana, F., Rosell, F., Graf, P. M., Williams, H., Gunner, R., Hopkins, L., Marks, N., Geraldi, N. R., Duarte, C. M., Scott, R., Strano, M. S., Robotka, H., Eizaguirre, C., Fahlman, A., Shepard, E. L. C., 2019. Estimates for energy expenditure in free living animals using acceleration proxies; a reappraisal. In Journal of Animal Ecology https://doi.org/10.1111/1365-2656.13040

Yousefpour, A., Fung, C., Nguyen, T., Kadiyala, K., Jalali, F., Niakanlahiji, A., Kong, J. and Jue, J. P. (2019). All one needs to know about fog computing and related edge computing paradigms: A complete survey. In Journal of Systems Architecture. Volume 98, September 2019. 289-330 https://doi.org/10.1016/j.sysarc.2019.02.009

Zhan, A., Mohan, S., Tarolli, C., Schneider, R. B., Adams, J. L., Sharma, S., Elson, M. J., Spear, K. L., Glidden, A. M., Little, M. A., Terzis, A., Dorsey, E. R., Saria, S., 2018. Using Smartphones and Machine Learning to Quantify Parkinson Disease Severity: The Mobile Parkinson Disease Score. In JAMA Neurolology 75(7):876-880. https://doi.org/10.1001/jamaneurol.2018.0809 